\title{
A Comprehensive Analysis of the Association of Psoas and Masseter Muscles with Traumatic Brain Injury Using Computed Tomography Anthropometry
}

\author{
Hang Joo Cho, ${ }^{1}$ Yunsup Hwang, ${ }^{2}$ Seiyun Yang, ${ }^{3}$ Maru Kim ${ }^{1}$ \\ Department of Trauma Surgery, Uijeongbu St. Mary's Hospital, College of Medicine, The Catholic University of Korea, Seoul, Korea \\ Department of Radiology, ${ }^{2}$ Uijeongbu St. Mary's Hospital, College of Medicine, The Catholic University of Korea, Seoul, Korea \\ Department of Neurourgery, ${ }^{3}$ Uijeongbu St. Mary's Hospital, College of Medicine, The Catholic University of Korea, Seoul, Korea
}

Objective : Psoas and masseter muscles are known markers of sarcopenia. However, the relative superiority of either muscle as a marker is unclear. Therefore, this study analyzed the two muscles in patients with a prognosis of traumatic brain injury (TBI).

Methods : Patients with TBI visiting a regional trauma center between January 2017 and December 2018 were selected, and their medical records were reviewed. TBI patients with an abbreviated injury score (AIS) of 4 or 5 were selected. Patients with an AIS of 4 or 5 at the chest, abdomen, and extremity were excluded. Patients with a hospital stay of 1 to 2 days were excluded. Both muscle areas were measured based on the initial computed tomography. The psoas muscle index (PMI) and the masseter muscle index (MMI) were calculated by dividing both muscle areas by height in meters squared $\left(\mathrm{cm}^{2} / \mathrm{m}^{2}\right)$. These muscle parameters along with other medical information were used to analyze mortality and the Glasgow outcome scale (GOS).

Results : A total of 179 patients, including 147 males (82.1\%), were analyzed statistically. The mean patient age was 58.0 years. The mortality rate was $16.8 \%$ (30 patients). The mean GOS score was 3.7. Analysis was performed to identify the parameters associated with mortality, which was a qualitative study outcome. The psoas muscle area ( $\left.16.9 \mathrm{vs.} 14.4 \mathrm{~cm}^{2}, p=0.028\right)$ and PMI $(5.9 \mathrm{vs}$. 5.1 $\mathrm{cm}^{2} / \mathrm{m}^{2}, p=0.004$ ) showed statistical differences between the groups. The PMI was also statistically significant as a risk factor for mortality in logistic regression analysis ( $p=0.023$; odds ratio, $0.715 ; 95 \%$ confidence interval, $0.535-0.954$ ). Quantitative analyses were performed with the GOS scores. Bivariate correlation analysis showed a statistically significant correlation between PMI and GOS scores (correlation coefficient, $0.168 ; p=0.003$ ). PMI ( $p=0.004$, variation inflation factor 1.001) was significant in multiple regression analysis. The masseter muscle area and MMI did not show significance in the study.

Conclusion : Larger PMI was associated with statistically significant improved survival and GOS scores, indicating its performance as a superior prognostic marker. Further analyses involving a larger number of patients, additional parameters, and more precise settings would yield a better understanding of sarcopenia and TBI.

Key Words : Traumatic brain injury $\cdot$ Psoas $\cdot$ Masseter muscle $\cdot$ Computed tomography $\cdot$ Prognosis.

- Received : January 4, $2021 \cdot$ Revised : February 25, 2021 •Accepted : March 10, 2021

- Address for reprints : Maru Kim

Department of Trauma Surgery, Uijeongbu St. Mary's Hospital, College of Medicine, The Catholic University of Korea, 271 Cheonbo-ro, Uijeongbu 11765, Korea Tel : +82-31-820-5266, Fax : +82-31-820-5378, E-mail : maru@catholic.ac.kr, ORCID : https://orcid.org/0000-0002-8973-5622

This is an Open Access article distributed under the terms of the Creative Commons Attribution Non-Commercial License (http://creativecommons.org/licenses/by-nc/4.0) which permits unrestricted non-commercial use, distribution, and reproduction in any medium, provided the original work is properly cited. 


\section{INTRODUCTION}

Sarcopenia (Greek 'sarx' or flesh + 'penia' or loss) is defined as a progressive and generalized skeletal muscle disorder ${ }^{5)}$. It is an important marker of nutritional and physical status and overall health $^{6,11)}$. It is currently recognized as a muscle disease and considered an independent condition based on the International Classification of Diseases, 10th clinical edition ${ }^{1,20)}$. Clinically, it is associated with cardiovascular, respiratory, and malignant diseases ${ }^{4,8,15}$. Sarcopenia is also associated with an individual's quality of life and mortality ${ }^{3,9)}$.

Among the many methods available to assess muscle status, the measurement of psoas muscle area (PMA) using computed tomography (CT) is well known. Reports have related PMA to mortality and the quality of life in several critical patients $^{7,10,19)}$. CT measurement of the masseter muscle area (MMA) is an alternative method to analyze muscle status. The masseter muscle is associated with nutritional disorders such as obesity and also with geriatric conditions ${ }^{14)}$. It is also associated with the patient's cerebrovascular prognosis ${ }^{16,17)}$. Hu et al. ${ }^{13)}$ reported that MMA was associated with early mortality in patients with severe traumatic brain injury (TBI).

Both PMA and MMA are proven prognostic markers in various diseases. However, evidence to suggest the relative superiority of each muscle as a prognostic marker is inadequate. Therefore, the present study was undertaken to compare the prognostic values of both markers including the odds ratio and correlation coefficient in inpatients with TBI. A comprehensive analysis of qualitative and quantitative parameters is presented.

\section{MATERIALS AND METHODS}

This retrospective study was approved by the Institutinal Review Board of Uijeongbu St. Mary's Hospital (UC20RISI0093), and the requirement for informed consent was waived. Patients with TBI visiting the regional trauma center between January 2017 and December 2018 were selected. Their medical information was gathered via retrospective chart review. General parameters including age, sex, height, weight, length of hospital stay, and trauma-related factors such as intubation, operation, Glasgow coma scale (GCS) score, abbreviated injury score (AIS), and injury severity score (ISS) were evaluated. Glasgow outcome scale (GOS) scores and mortality were analyzed together to determine the clinical outcomes. Patients with endotracheal intubation and scoring 8 or less on the GCS were regarded as having a severe TBI and compared to those with scores of 9 or higher on the GCS. Patient body mass index (BMI) was calculated and those with a BMI higher than 25 were regarded as obese.

Initially, CT was used to perform the muscle measurements. Common sites were used for the anthropometric studies, as described previously. The cross-sectional area of the psoas muscle was measured at the level of the third lumbar vertebral body bilaterally as the PMA ${ }^{12}$. The cross-sectional area of the masseter muscle was measured $2 \mathrm{~cm}$ below the zygomatic arch bilaterally as the MMA ${ }^{18)}$. The psoas muscle index (PMI) and the masseter muscle index (MMI) were calculated by dividing both muscle areas by height in meters squared $\left(\mathrm{cm}^{2} / \mathrm{m}^{2}\right)$. Patients with proper CT images were included in the study. Patients with tilted CT images or image artifacts were excluded. Patients with injured muscles or nearby bony structures were also excluded.

Patients with major TBI were selected based on an AIS of 4 or 5. Patients with an AIS of 4 or 5 at the chest, abdomen, or extremity were excluded to reduce bias associated with other conditions. Patients younger than 19 years and those with missing data were also excluded. Additionally, patients with a hospital stay of fewer than 2 days were excluded.

Comprehensive analyses were planned. Mortality was used as a qualitative outcome and GOS scores were used as a quantitative outcome. Initially, the patients were separated into two groups, survival and mortality, and their medical records were analyzed. An independent t-test was used for quantitative analysis, and chi-squared tests were used for qualitative analysis. Logistic regression analysis was performed to verify the risk factors. Bivariate correlation analysis of both muscle indices and GOS scores was conducted. Correlation coefficients were calculated after adjustment for age, BMI, AIS, and ISS. Additionally, multiple regression analysis was performed. All statistical analyses were performed using IBM SPSS Statistics for Windows, version 25.0 (IBM Corp., Armonk, NY, USA).

\section{RESULTS}

A total of 357 TBI patients had an AIS score of 4-5. Among 
them, 263 patients were suspected of having major trauma and underwent both brain and torso CT (chest + abdominopelvic CT). Two hundred and forty-three patients with proper CT images and 226 patients without injury to the chest or abdomen, or extremity AIS scores of $4-5$, were selected. Twenty patients were excluded due to age, and seven patients were excluded due to missing data. Additionally, 20 patients were excluded for missing data. The patient selection process is summarized in Fig. 1.

A total of 179 patients were finally included in the statistical analyses. The mean patient age was 58.0 years. The study included 147 male patients (82.1\%). The patient population was as follows : 51 patients with obesity (28.5\%), 64 patients with severe TBI (35.8\%), and 92 patients treated with neurosurgery (51.4\%). The mean PMA and MMA values were $16.5 \mathrm{~cm}^{2}$ and $9.8 \mathrm{~cm}^{2}$, respectively. The mean PMI and MMI values were 5.8 and $3.5 \mathrm{~cm}^{2} / \mathrm{m}^{2}$, respectively. Mortality was recorded in $30 \mathrm{pa}-$ tients (16.8\%). The mean GOS score was 3.7. Other basic information is summarized in Table 1.

The parameters related to mortality, which was a qualitative study outcome, were analyzed. PMA (16.9 vs. $14.4 \mathrm{~cm}^{2}$, $p=0.028$ ) and PMI (5.9 vs. $5.1 \mathrm{~cm}^{2} / \mathrm{m}^{2}, p=0.004$ ) showed statistically significant differences between the survival and mortality groups, as did patients with endotracheal intubation and scoring 8 or less on the GCS $(p<0.001)$. Also, the external and

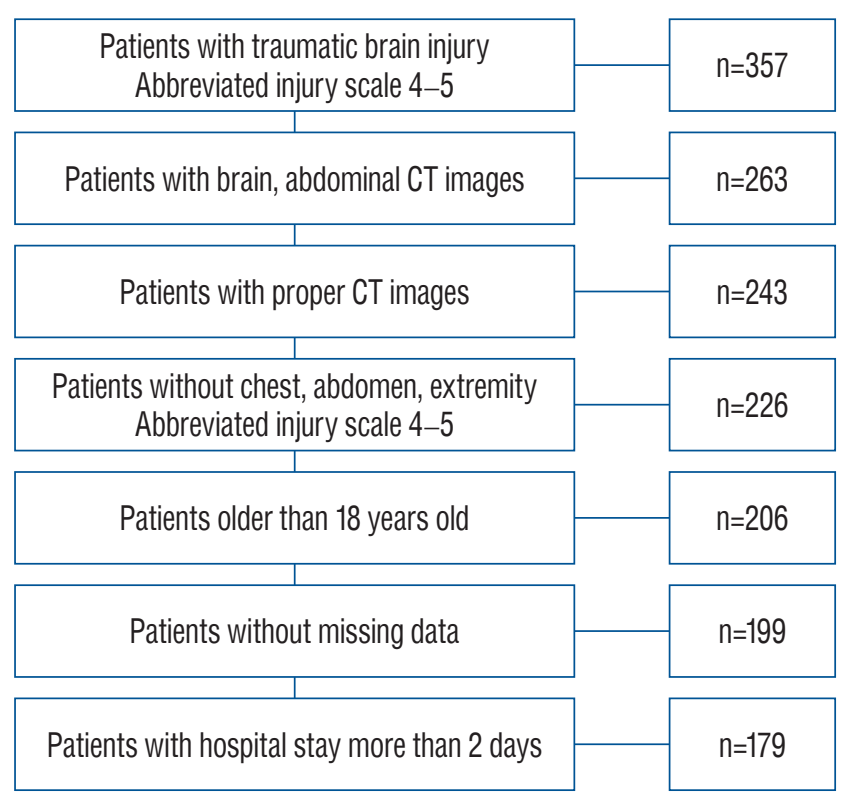

Fig. 1. Flowchart showing the enrollment of patients with traumatic brain injury. CT : computed tomography. facial AIS values differed statistically. The mortality analysis is summarized in Table 2. A logistic regression analysis of mor-

Table 1. Patients' baseline demographics

\begin{tabular}{lc}
\hline & \multicolumn{1}{c}{ Value } \\
\hline Age (years) & $57.95 \pm 16.24$ \\
Sex & $32(17.9)$ \\
Female & $147(82.1)$ \\
Male & $167.59 \pm 8.22$ \\
Height $(\mathrm{cm})$ & $65.65 \pm 11.32$ \\
Weight $(\mathrm{kg})$ & $23.27 \pm 3.03$ \\
Body mass index $\left(\mathrm{kg} / \mathrm{cm}^{2}\right)$ & \\
Obesity & $128(71.5)$ \\
No & $51(28.5)$ \\
Yes
\end{tabular}

Traumatic brain injury

Non-severe

$115(64.2)$

Severe

64 (35.8)

\begin{tabular}{lc} 
Operation & \\
No & $87(48.6)$ \\
Yes & $92(51.4)$ \\
Psoas muscle area $\left(\mathrm{cm}^{2}\right)$ & $16.47 \pm 5.60$ \\
Masseter muscle area $\left(\mathrm{cm}^{2}\right)$ & $9.76 \pm 2.50$ \\
Psoas muscle index $\left(\mathrm{cm}^{2} / \mathrm{m}^{2}\right)$ & $5.80 \pm 1.75$ \\
Masseter muscle index $\left(\mathrm{cm}^{2} / \mathrm{m}^{2}\right)$ & $3.47 \pm 1.75$ \\
Abbreviated injury score & \\
Head and neck & $4.54 \pm 5.0$ \\
Face & $0.36 \pm 0.76$ \\
Chest & $1.03 \pm 1.35$ \\
Abdomen & $0.25 \pm 0.75$ \\
Extremity & $0.75 \pm 1.08$ \\
External & $0.36 \pm 0.54$ \\
Injury severity score & $26.59 \pm 6.43$ \\
Glasgow outcome score & $3.65 \pm 1.50$ \\
1 & $30(16.8)$ \\
2 & $9(5.0)$ \\
Mortality & $35(19.6)$ \\
No & $24(13.4)$ \\
\hline & $81(45.3)$ \\
\hline & $149(83.2)$ \\
\hline & $30(16.8)$ \\
\hline & \\
\hline & \\
\hline
\end{tabular}

Values are presented as mean \pm standard deviation or number (\%) 
tality parameters is presented in Table 3. Patients with endotracheal intubation and scoring 8 or less on the GCS was statistically associated with mortality. PMI was also a statistically significant risk factor for mortality $(B=-0.336$; $p=0.023$; odds ratio, $0.715 ; 95 \%$ confidence interval, $0.535-0.954)$. The mean MMA and MMI decreased in the mortality group, but the difference was not significant.

Analysis of the GOS scores was performed and the results

Table 2. Mortality analysis of patients with traumatic brain injury

\begin{tabular}{|c|c|c|c|}
\hline Parameter & Survival $(n=149,83.2 \%)$ & Mortality $(n=30,16.8 \%)$ & $p$-value \\
\hline Age (years) & $57.23 \pm 16.59$ & $61.53 \pm 14.08$ & 0.186 \\
\hline \multicolumn{4}{|l|}{ Sex } \\
\hline Female & $24(75.0)$ & $8(25.0)$ & 0.168 \\
\hline Male & $125(85.0)$ & $22(15.0)$ & \\
\hline Height $\left(\mathrm{cm}^{2}\right)$ & $167.76 \pm 8.07$ & $166.73 \pm 9.04$ & 0.535 \\
\hline Weight (kg) & $65.76 \pm 11.23$ & $65.08 \pm 11.96$ & 0.767 \\
\hline Body mass index $\left(\mathrm{kg} / \mathrm{m}^{2}\right)$ & $23.27 \pm 3.08$ & $23.27 \pm 2.85$ & 0.999 \\
\hline \multicolumn{4}{|l|}{ Obesity } \\
\hline No & $106(82.8)$ & $22(17.2)$ & 0.808 \\
\hline Yes & $43(84.3)$ & $8(15.7)$ & \\
\hline \multicolumn{4}{|l|}{ Severity } \\
\hline $\mathrm{GCS} \geq 9$ & $108(93.9)$ & $7(6.1)$ & $<0.001$ \\
\hline GCS $\leq 8$ or intubated & $41(64.1)$ & $23(35.9)$ & \\
\hline \multicolumn{4}{|l|}{ Operation } \\
\hline No & $77(88.5)$ & $10(11.5)$ & 0.067 \\
\hline Yes & $72(78.3)$ & $20(21.7)$ & \\
\hline Psoas muscle area $\left(\mathrm{cm}^{2}\right)$ & $16.88 \pm 5.70$ & $14.43 \pm 4.65$ & 0.028 \\
\hline Masseter muscle area $\left(\mathrm{cm}^{2}\right)$ & $9.79 \pm 2.54$ & $9.59 \pm 2.32$ & 0.694 \\
\hline Psoas muscle index $\left(\mathrm{cm}^{2} / \mathrm{m}^{2}\right)$ & $5.94 \pm 1.80$ & $5.10 \pm 1.27$ & 0.004 \\
\hline Masseter muscle index $\left(\mathrm{cm}^{2} / \mathrm{m}^{2}\right)$ & $3.48 \pm 0.87$ & $3.45 \pm 0.80$ & 0.883 \\
\hline \multicolumn{4}{|l|}{ Abbreviated injury score } \\
\hline Head and neck & $4.53 \pm 0.50$ & $4.60 \pm 0.50$ & 0.487 \\
\hline Face & $0.40 \pm 0.80$ & $0.17 \pm 0.46$ & 0.030 \\
\hline Chest & $1.03 \pm 1.34$ & $1.03 \pm 1.40$ & 0.981 \\
\hline Abdomen & $0.23 \pm 0.72$ & $0.33 \pm 0.88$ & 0.513 \\
\hline Extremity & $0.77 \pm 1.08$ & $0.70 \pm 1.12$ & 0.765 \\
\hline External & $0.40 \pm 0.54$ & $0.17 \pm 0.46$ & 0.020 \\
\hline Injury severity score & $26.52 \pm 6.33$ & $26.90 \pm 7.00$ & 0.771 \\
\hline
\end{tabular}

Values are presented as mean \pm standard deviation or number (\%). An independent t-test was used for quantitative analysis, and chi-squared tests were used for qualitative analysis. GCS : Glasgow coma score

Table 3. Results of logistic regression analysis of mortality parameters

\begin{tabular}{lccc}
\hline Parameter & B & p-value & OR (95\% Cl) \\
\hline Psoas muscle index & -0.336 & 0.023 & $0.715(0.535-0.954)$ \\
Glasgow coma score $\leq 8$ or intubated & -2.185 & $<0.001$ & $0.112(0.044-0.287)$ \\
\hline
\end{tabular}

OR: odds ratio, $\mathrm{Cl}$ : confidence interval 
Table 4. Results of bivariate and partial correlation analyses with Glasgow outcome scale

\begin{tabular}{lcc}
\hline Type of analysis & Psoas muscle index & Masseter muscle index \\
\hline Bivariate correlation analysis & & \\
Kendall's tau b & 0.168 & 0.061 \\
$\quad$ Correlation coefficient & 0.003 & 0.281 \\
$p$-value & & 0.067 \\
Partial correlation analysis & 0.176 & 0.390 \\
Adjustment with age, BMI, AIS, ISS & 0.023 & 0.106 \\
Correlation coefficient & & 0.170 \\
$p$-value & 0.211 & 0.006 \\
Adjustment with BMI, AIS, ISS & & 0.060 \\
Correlation coefficient & 0.211 & 0.434 \\
$p$-value & 0.005 & \\
Adjustment with AIS, ISS & & \\
$\quad$ Correlation coefficient & & \\
$p$-value & & \\
\hline
\end{tabular}

BMI : body mass index, AIS : abbreviated injury score, ISS : injury severity score

Table 5. Result of multiple regression analysis with Glasgow outcome scale

\begin{tabular}{lccc}
\hline Parameter & B & p-value & Variation inflation factor \\
\hline Psoas muscle index & 0.184 & 0.004 & 1.001 \\
AIS - external & 0.476 & 0.020 & 1.001 \\
\hline
\end{tabular}

AIS : abbreviated injury score

were defined as a quantitative outcome. Bivariate correlation analysis showed a statistical correlation between PMI and GOS (correlation coefficient, 0.168; $p=0.003$ ). Partial correlation analyses were performed after adjustment for age, BMI, AIS, and ISS. PMI was correlated with GOS scores after multiple adjustments, but the MMI was not significantly correlated (Table 4). The results of the multiple regression analysis are presented in Table $5\left(\mathrm{R}^{2}=0.048\right)$. PMI showed a statistically significant association with $\mathrm{GOS}(\mathrm{B}=0.184, p=0.004$, variation inflation factor 1.001).

\section{DISCUSSION}

The present study is unique for several reasons. First, the study goal was uncommon in that it was planned to compare the relative superiority of the psoas muscle (PM) and masseter muscle (MM), which are well-known prognostic markers.

Second, the study selected patients with severe TBI (AIS 4 or 5). Patients with other severe injuries were excluded. To reduce the impact of other injuries, patients with AIS scores of 4 or 5 at the chest, abdomen, or extremity were excluded. Additionally, patients with hospital stays of longer than 2 days were selected. Dead trauma patients during this period usually had severe, incurable injuries and their mortality might be inevitable. In addition, the guardians of some patients wanted patients transferred to another hospital. These patients were regarded as improper candidates for the analysis and excluded from the study. Consequently, the study included patients with serious TBI and reduced the possibility of bias, although the number of analyzed patients was decreased.

Third, comprehensive analyses of qualitative and quantitative parameters were planned. A similar study of PM and MM analysis was reported previously ${ }^{21)}$. Both parameters were analyzed based on mortality alone, which was a qualitative parameter. However, this study analyzed mortality and GOS scores comprehensively.

The AIS face and external scores showed a statistical differ- 
ence. However, the mean AIS score was less than 1 point, indicating a very minor injury. Those injuries were too minor and might have had an insignificant effect on mortality or GOS scores.

However, the authors could not define or analyze sarcopenia in the present study. The initial target for analysis was not sarcopenia but involved both the PM and MM. Muscle strength and physical performance were not assessed because patient cooperation was hindered by their TBI. Further, it has been reported that sarcopenia cannot be represented by a single muscle ${ }^{2}$. Nevertheless, the relationship between both muscles and the prognosis of patients with TBI was determined statistically. Further study is needed to diagnose sarcopenia in a larger population of patients.

\section{CONCLUSION}

A larger PMA was associated with statistically significant survival outcomes and better GOS scores and showed superiority as a prognostic marker. Although TBI severity was the most significant mortality parameter, PMI was statistically significant in various analyses, suggesting that the PMI can be used to predict the prognosis of patients with TBI, whereas the MM showed no statistically significant results and was not comparable to the PM. Consequently, the PM showed its superiority over MM as a prognostic marker for TBI. Further analyses with a larger number of patients might show different results because this study had a relatively low number of participants. Additional parameters and more precise settings can yield better outcomes and increase our understanding of the relationship between sarcopenia and TBI.

\section{CONFLICTS OF INTEREST}

No potential conflict of interest relevant to this article was reported.

\section{INFORMED CONSENT}

This type of study does not require informed consent.

\section{AUTHOR CONTRIBUTIONS}

\author{
Conceptualization : HJC \\ Data curation : YH, SY, MK \\ Formal analysis: MK \\ Funding acquisition : $\mathrm{MK}$ \\ Methodology : HJC, MK \\ Visualization : YH, SY, HJC \\ Writing - original draft : MK \\ Writing - review \& editing : HJC
}

\section{ORCID}

$\begin{array}{ll}\text { Hang Joo Cho } & \text { https://orcid.org/0000-0003-3322-744X } \\ \text { Yunsup Hwang } & \text { https://orcid.org/0000-0002-6827-6463 } \\ \text { Seiyun Yang } & \text { https://orcid.org/0000-0001-8431-3781 } \\ \text { Maru Kim } & \text { https://orcid.org/0000-0002-8973-5622 }\end{array}$

\section{- Acknowledgements}

The authors wish to acknowledge the financial support of the Catholic Medical Center Research Foundation made in the program year of 2019 .

\section{References}

1. Anker SD, Morley JE, von Haehling $S$ : Welcome to the ICD-10 code for sarcopenia. J Cachexia Sarcopenia Muscle 7 : 512-514, 2016

2. Baracos VE : Psoas as a sentinel muscle for sarcopenia: a flawed premise. J Cachexia Sarcopenia Muscle 8: 527-528, 2017

3. Beaudart C, Biver E, Reginster JY, Rizzoli R, Rolland Y, Bautmans I, et

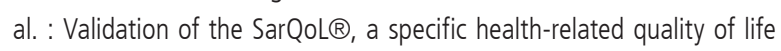
questionnaire for Sarcopenia. J Cachexia Sarcopenia Muscle 8 : 238-244, 2017

4. Bone $A E$, Hepgul $N$, Kon $S$, Maddocks $M$ : Sarcopenia and frailty in chronic respiratory disease. Chron Respir Dis 14 : 85-99, 2017

5. Cruz-Jentoft AJ, Bahat G, Bauer J, Boirie Y, Bruyère O, Cederholm T, et al. : Sarcopenia: revised European consensus on definition and diagnosis. Age Ageing 48 : 16-31, 2019

6. Cruz-Jentoft AJ, Sayer AA : Sarcopenia. Lancet 393 : 2636-2646, 2019

7. Damluji AA, Rodriguez G, Noel T, Davis L, Dahya V, Tehrani B, et al. : Sarcopenia and health-related quality of life in older adults after transcatheter aortic valve replacement. Am Heart J 224 : 171-181, 2020

8. Davis MP, Panikkar $\mathrm{R}$ : Sarcopenia associated with chemotherapy and targeted agents for cancer therapy. Ann Palliat Med 8 : 86-101, 2019 
9. De Buyser SL, Petrovic M, Taes YE, Toye KR, Kaufman JM, Lapauw B, et al. : Validation of the FNIH sarcopenia criteria and SOF frailty index as predictors of long-term mortality in ambulatory older men. Age Ageing45 : 602-608, 2016

10. Drudi LM, Phung K, Ades M, Zuckerman J, Mullie L, Steinmetz OK, et al. : Psoas muscle area predicts all-cause mortality after endovascular and open aortic aneurysm repair. Eur J Vasc Endovasc Surg 52 : 764769, 2016

11. Gilligan LA, Towbin AJ, Dillman JR, Somasundaram E, Trout AT : Quantification of skeletal muscle mass: sarcopenia as a marker of overall health in children and adults. Pediatr Radiol 50 : 455-464, 2020

12. Hamaguchi $Y$, Kaido T, Okumura S, Kobayashi A, Hammad A, Tamai Y, et al. : Proposal for new diagnostic criteria for low skeletal muscle mass based on computed tomography imaging in Asian adults. Nutrition 32 : 1200-1205, 2016

13. Hu P, Uhlich R, White J, Kerby J, Bosarge P : Sarcopenia measured using masseter area predicts early mortality following severe traumatic brain lijury. J Neurotrauma 35 : 2400-2406, 2018

14. Hwang Y, Lee YH, Cho DH, Kim M, Lee DS, Cho HJ : Applicability of the masseter muscle as a nutritional biomarker. Medicine (Baltimore) 99 : e19069, 2020

15. Lee SJ, Park YJ, Cartmell KB : Sarcopenia in cancer survivors is associated with increased cardiovascular disease risk. Support Care Cancer
$26: 2313-2321,2018$

16. Lindström I, Protto S, Khan N, Hernesniemi J, Sillanpää N, Oksala N : Association of masseter area and radiodensity with three-month survival after proximal anterior circulation occlusion. J Neurointerv Surg 13 : 25-29, 2021

17. Oksala NKJ, Lindström I, Khan N, Pihlajaniemi VJ, Lyytikäinen LP, Pienimäki JP, et al. : Pre-operative masseter area is an independent predictor of long-term survival after carotid endarterectomy. Eur J Vasc Endovasc Surg $57: 331-338,2019$

18. Tanabe C, Reed MJ, Pham TN, Penn K, Bentov I, Kaplan SJ : Association of brain atrophy and masseter sarcopenia with 1-year mortality in older trauma patients. JAMA Surg 154 : 716-723, 2019

19. Toptas M, Yalcin M, Akkoc I, Demir E, Metin C, Savas Y, et al. : The relation between sarcopenia and mortality in patients at intensive care unit. Biomed Res Int 2018 : 5263208, 2018

20. Vellas B, Fielding RA, Bens C, Bernabei R, Cawthon PM, Cederholm T, et al. : Implications of ICD-10 for sarcopenia clinical practice and clinical trials: report by the international conference on frailty and sarcopenia research task force. J Frailty Aging 7 : 2-9, 2018

21. Wallace JD, Calvo RY, Lewis PR, Brill JB, Shackford SR, Sise MJ, et al. : Sarcopenia as a predictor of mortality in elderly blunt trauma patients: comparing the masseter to the psoas using computed tomography. J Trauma Acute Care Surg 82 : 65-72, 2017 